\title{
Mandibular Osteonecrosis Associated with Antacid Therapy (Esomeprazole)
}

\author{
Simone Marconcini, Enrica Giammarinaro, Saverio Cosola, Anna Maria Genovesi, Ugo Covani \\ Tuscan Stomatologic Institute, Versilia General Hospital, Lido di Camaiore, Italy
}

Received: 19/09/2019

Accepted: 23/09/2019

Published: 14/10/2019

How to cite this article: Marconcini S, Giammarinaro E, Cosola S, Genovesi AM, Covani U. Mandibular osteonecrosis associated with antacid therapy (esomeprazole). EJCRIM 2019;6: doi:10.12890/2019_001279.

Conflicts of Interests: The Authors declare that there are no competing interest

This article is licensed under a Commons Attribution Non-Commercial 4.0 License

\section{ABSTRACT}

Introduction: Osteonecrosis of the jaw has been consistently reported in the literature associated tothe high-dose intravenous bisphosphonate therapy. However, osteonecrosis can also occur in patients who have other risk factors.

Case description: An unusual case of ONJ in a patient being treated with esomeprazole is reported.

Discussion: The probable association between proton pump inhibitor intake and osteonecrosis of the jaw should alert clinicians. Collaborations between medical and dental doctor and an early diagnosis might prevent or reduce the morbidity resulting from advanced destructive lesions of the jaw bone.

\section{LEARNING POINTS}

- Osteonecrosis of the jaw (ONJ) can occur in patients treated with bisphosphonates and corticosteroids and is associated with oral surgical procedures involving bone.

- Antacid drugs commonly used to treat gastro-oesophageal reflux could affect bone metabolism although no cases of ONJ in patients using proton pump inhibitors have been reported.

- Medical and dental practitioners should collaborate to prevent ONJ, identify previously unreported drug interactions, and treat patients in a more comprehensive manner.

\section{KEYWORDS}

Mandibular osteonecrosis; antacid therapy; esomeprazole; osteonecrosis of the jaw; proton pump inhibitors.

\section{INTRODUCTION}

Osteonecrosis of the jaw (ONJ) has been consistently reported in the literature since the 19th century ${ }^{[1]}$. It is an uncommon clinical entity with several causes, including head and neck irradiation, chemotherapy, trauma and periodontal disease ${ }^{[2]}$. Over the last 10 years, an increasing number of case reports have suggested that high-dose intravenous bisphosphonates may be associated with ONJ[3]. Patients typically presented with pain, impaired healing of extraction sockets, or exposed bone. Nearly all patients (86\%) had undergone dental procedures and most were receiving long-term chemotherapy and short-term intermittent corticosteroid treatment ${ }^{[3]}$. However, ONJ can also occur in patients who are not treated with bisphosphonates and in patients without the traditional risk factors ${ }^{[4]}$. The authors report an unusual case of $\mathrm{ONJ}$ in a patient being treated with esomeprazole. 


\section{CASE DESCRIPTION}

A72-year-old man was referred to the Istituto Stomatologico Toscano (Lido di Camaiore, Italy) in September 2017. The patient had a complete removable prosthesis in the lower jaw and a partial removable prosthesis in the upper jaw. He complained of a chronic painful swelling in the left mandibular area. His medical history revealed intake of docetaxel and the steroid prednisone over a 3-year period following a diagnosis of prostate cancer. Extra-oral examination did not reveal any facial asymmetry. However, on intra-oral examination, an ulcerated area in the left lower jaw was noted after the prosthesis was removed (Fig. 1). The ulcer was exposing necrotic bone at the level of the alveolar crest. The lesion measured approximately $2 \mathrm{~cm}$ (mesiodistal) by $1 \mathrm{~cm}$ (buccal-lingual). Radiographic evaluation showed radiolucency in the corresponding area (Fig. 2). A provisional diagnosis of ONJ was made. After discussion with the patient, surgical debridement of the pathological lesion was performed under local anaesthesia (2\% mepivacaine), with scraping instruments and a bone cutting forceps (Fig. 3 ). The mouth of the patient was rinsed for 1,5 minute with ozonized water (Aquolab EB2C Srl), for decontamination purposes, prior to the surgery and amoxicillin (500 mg three times a day for 7 days) and metronidazole (500 mg three times a day for 7 days) were prescribed to start the therapy before surgery. However, 3 months after surgery, the lesion had not healed and the patient was complaining of a moderate ache at the site of intervention.

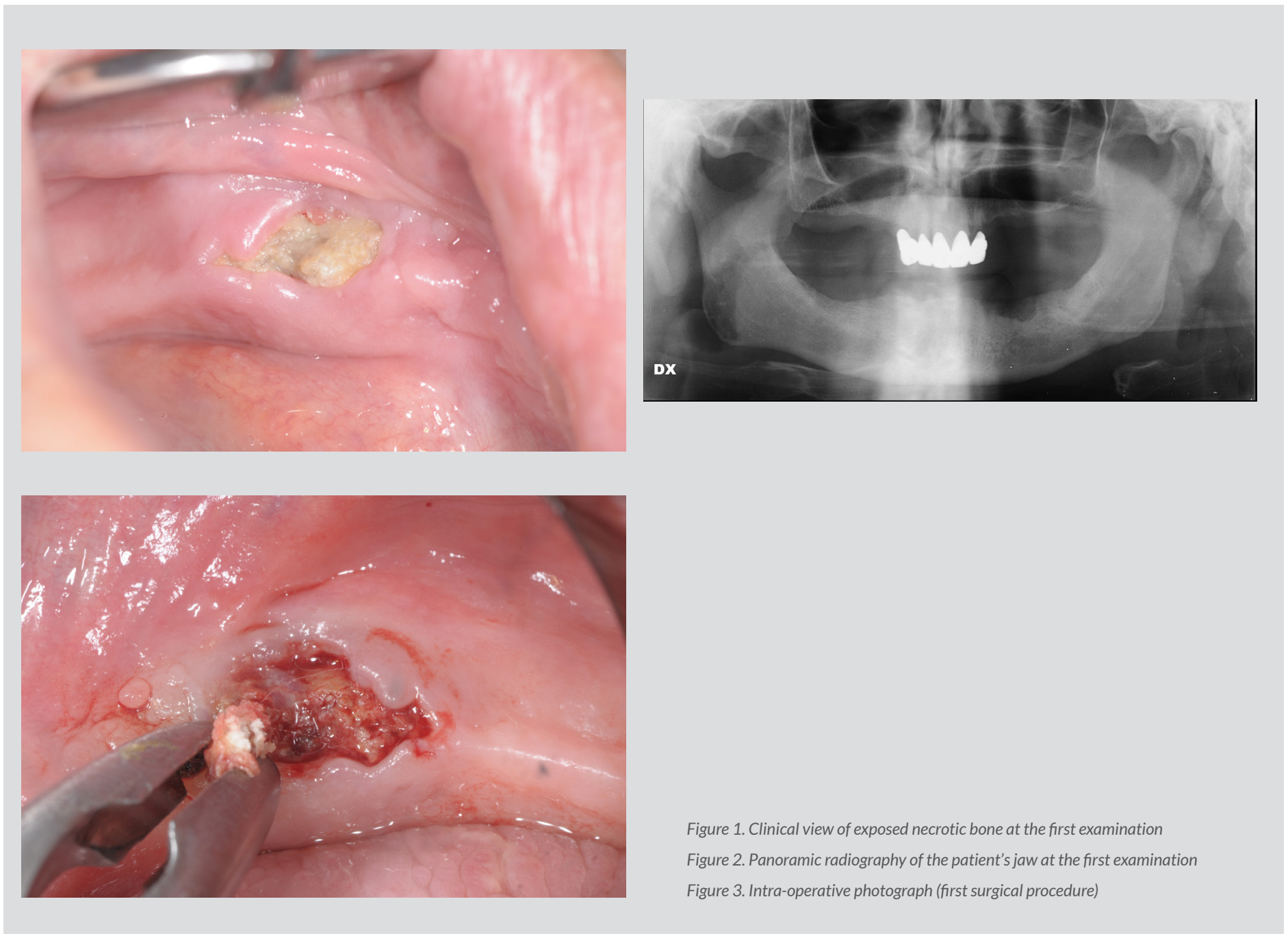

It was decided to reconsider the possible side-effects and interactions of the drugs currently used by the patient. Further enquiry revealed that the patient was a long-term user of an over-the-counter proton pump inhibitor (PPI; Nexium, AstraZeneca, Basiglio, Milan, Italy) for the symptomatic treatment of gastro-oesophageal reflux. PPIs interfere with bone metabolism, as acknowledged by the FDA. It was therefore decided to immediately stop the antacid drug and to excise the necrotic bone, with scraping of the affected area in order to reach healthy 
bleeding bone (Fig. 4). The necrotic area was treated with local ozone therapy (Ozone DTA, Sweden \& Martina S.p.A.) 2 days before surgery, and then once a day for the first week after surgery, followed by once a week for the next 2 months. Histological analysis of the excised bone confirmed the diagnosis of ONJ. A second cycle of antibiotic therapy was prescribed (amoxicillin 500 mg four times a day for 5 days) with chlorhexidine gluconate $0.2 \%$ for mouth rinsing. The patient's symptoms resolved and the soft tissues had healed completely after 2 months (Fig. 5). The patient returned for follow-up visits at 6 months and 12 months with no adverse events recorded.

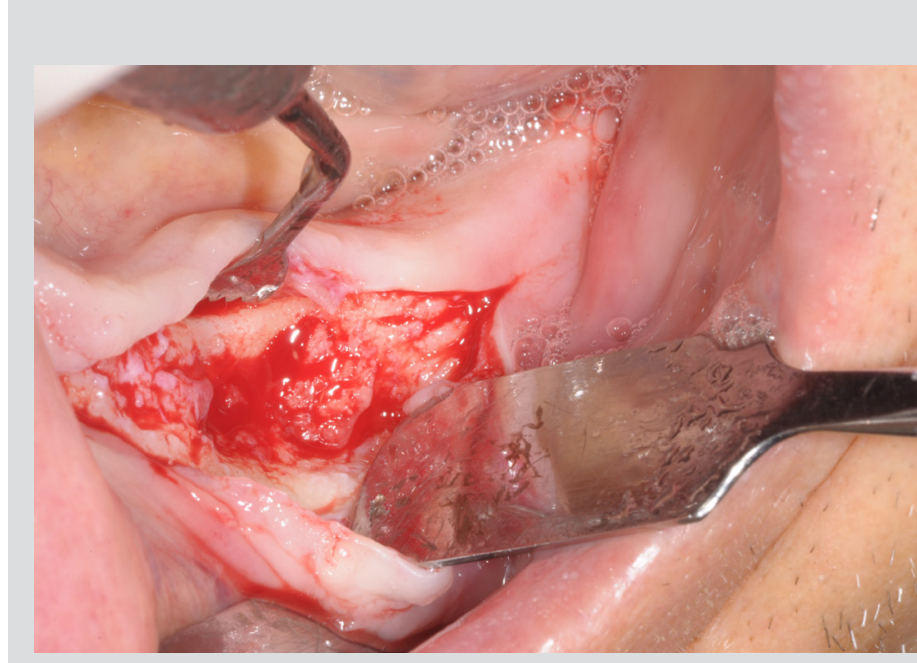

Figure 4. Intra-operative photograph (second surgical procedure)

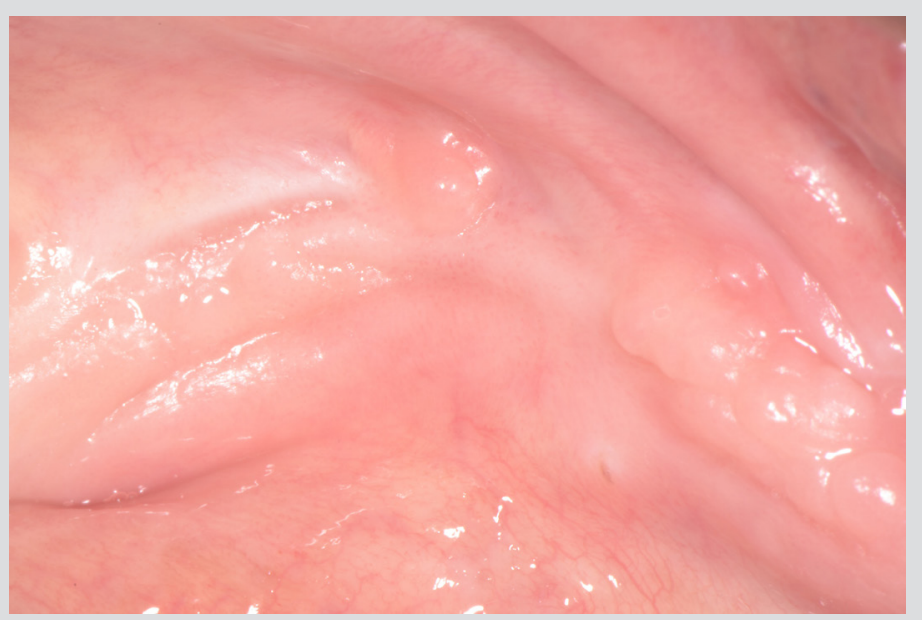

Figure 5. Healed tissues 2 months after the second surgical procedure and the suspension of proton pump inhibitor therapy

\section{DISCUSSION}

PPIs are commonly prescribed worldwide ${ }^{[5]}$ and frequently used to treat gastro-intestinal disorders such as acid reflux and ulcers. They suppress the functions of the proton pump (H1/K1 ATPase), thus inhibiting gastric acidity ${ }^{[6]}$. Adverse effects, such as increased risk of infections, interaction with other drugs, dementia and reduced mineral absorption have been reported ${ }^{[7,8]}$. Reports have also shown that PPIs can impair bone metabolism: (a) the suppression of gastric acidity results in a reduction in intestinal calcium uptake and (b) PPIs downregulate osteoclastic activity by inhibiting their surface proton pumps ${ }^{[9]}$.

Our patient presented with jaw osteonecrosis which resolved after suspension of antacid therapy with esomeprazole (Nexium). A recent cohort study, which included over 60,000 alendronate users in Denmark, investigated the presence of potential risk factors for ONJ requiring surgical treatment ${ }^{[10]}$. The authors suggested that $\mathrm{ONJ}$ risk appeared to be higher in patients with concomitant risk factors other than bisphosphonate treatment: a history of rheumatoid disease and the use of PPIs were independently associated with surgically treated ONJ. The esomeprazole Nexium is one of the most commonly used PPIs and it is often taken together with non-steroidal anti-inflammatory drugs (NSAIDs). Furthermore, NSAIDs are frequently prescribed for pain control after oral surgery, and their usage is also related to bone health ${ }^{[11]}$. In brief, adult patients who have a positive history of bisphosphonate use, cancer and oral surgery might be more likely to use PPIs and NSAIDs, and, therefore, be more prone to ONJ.

Recent cohort studies on dental implant outcomes have suggested that PPI intake is associated with an increased risk of implant failure [12]. This association persisted after adjustment for multiple confounding factors ${ }^{[13]}$. Furthermore, study participants in implant programs had not included patients treated with bisphosphonates, since this was one of the exclusion criteria ${ }^{[14]}$.

Long-term use of PPIs is associated with reduced serum levels of calcium and vitamin B12, impaired collagen deposition in the extracellular matrix, and reduced bone strength. Recent evidence has also supported a gene modulating effect by PPIs towards lower expression of bone formation markers.

The observation of an association between PPI intake and ONJ should alert practitioners ${ }^{[15]}$. An early diagnosis might prevent or reduce the morbidity resulting from advanced destructive lesions of the jaw bone. Furthermore, osteoclast-selective PPIs may be an interesting anti-resorptive agent for future study. 


\section{REFERENCES}

1. Kumar SK, Meru M, Sedghizadeh PP. Osteonecrosis of the jaws secondary to bisphosphonate therapy: a case series. J Contemp Dent Pract 2008;9:63-69.

2. Advisory Task Force on Bisphosphonate-Related Osteonecrosis of the Jaws, American Association of Oral and Maxillofacial Surgeons. American Association of Oral and Maxillofacial Surgeons position paper on bisphosphonate-related osteonecrosis of the jaws. J Oral Maxillofac Surg 2007;65:369-376.

3. Reilly MM, Moore S. Osteonecrosis of the jaw. Oncology (Williston Park) 2008;22:39-41.

4. Junquera L, Gallego L. Nonexposed bisphosphonate-related osteonecrosis of the jaws: another clinical variant? J Oral Maxillofac Surg 2008 ;66:1516-1517.

5. Ali T, Roberts, DN, Tierney WM. Long-term safety concerns with proton pump inhibitors. Am J Med 2009;10:896-903.

6. Stedman C, Barclay M. Review article: comparison of the pharmacokinetics, acid suppression and efficacy of proton pump inhibitors. Aliment Pharmacol Ther 2000;14:963-978.

7. Wright MJ, Proctor DD, Insogna KL, Kerstetter JE. Proton pump-inhibiting drugs, calcium homeostasis, and bone health. Nutr Rev 2008;66:103-108.

8. Eusebi LH, Rabitti S, Artesiani ML, et al. Proton pump inhibitors: risks of long-term use. J Gastroenterol Hepatol 2017;32:1295-1302.

9. O'Connell MB, Madden DM, Murray AM, et al. Effects of proton pump inhibitors on calcium carbonate absorption in women: a randomized crossover trial. Am J Med 2005;118:778-781.

10. Eiken A, Prieto-Alhambra, D, Eastell R, et al. Surgically treated osteonecrosis and osteomyelitis of the jaw and oral cavity in patients highly adherent to alendronate treatment: a nationwide user-only cohort study including over 60,000 alendronate users. Osteoporos Int 2017;28:2921-2928.

11. Zaidi M. Modularity of osteoclast behaviour and "mode-specific" inhibition of osteoclast function. Biosci Rep 1990;10:547-556.

12. Wu X, Al-Abedalla K, Abi-Nader S, et al. Proton pump inhibitors and the risk of osseointegrated dental implant failure: a cohort study. Clin Implant Dent Relat Res 2017;19:222232.

13. Nicolatou-Galitis O, Kouri M, Papadopoulou E, et al.; MASCC Bone Study Group. Osteonecrosis of the jaw related to non-antiresorptive medications: a systematic review. Support Care Cancer 2019;27(2):383-394.

14. Chrcanovic BR, Kisch J, Albrektsson T, et al. Intake of proton pump inhibitors is associated with an increased risk of dental implant failure. Int J Oral Maxillofac Implants 2017:32:1097-1102.

15. Ruiz AC, Carrascosa MF, Concha ST, Gil AH, Rivero JG. Interstitial lung disease in a patient treated with denosumab. Eur J Case Rep Intern Med 2019;6(7):001131. 